\title{
Wave Harmonization in Hierarchic Quasicrystals by the Analytic Metric
}

\author{
Antony J. Bourdillon \\ UHRL, San Jose, CA, USA \\ Email: bourdillona@sbcglobal.net
}

How to cite this paper: Bourdillon, A.J. (2019) Wave Harmonization in Hierarchic Quasicrystals by the Analytic Metric. Journal of Modern Physics, 10, 1364-1373. https://doi.org/10.4236/jmp.2019.1011090

Received: September 10, 2019

Accepted: October 18, 2019

Published: October 21, 2019

Copyright $\odot 2019$ by author(s) and Scientific Research Publishing Inc. This work is licensed under the Creative Commons Attribution International License (CC BY 4.0).

http://creativecommons.org/licenses/by/4.0/

\begin{abstract}
Thirty seven years after the discovery of quasicrystals, their diffraction is completely described by harmonization between the sine wave probe with hierarchic translational symmetry in a structure that is often called quasiperiodic. The diffraction occurs in geometric series that is a special case of the Fibonacci sequence. Its members are irrational. When substitution is made for the golden section $\tau$ by the semi-integral value 1.5 , a coherent set of rational numbers maps the sequence. Then the square of corresponding ratios is a metric that harmonizes the sine wave probe with the hierarchic structure, and the quasi-Bragg angle adjusts accordingly. From this fact follows a consistent description of structure, diffraction and measurement.
\end{abstract}

\section{Keywords}

Quasicrystal, Icosahedra, Hierarchic, Harmonization, Metric, Geometric Series

\section{Introduction}

"Physical" theories degenerate easily to common myth when the basic norms of physical practice are ignored. These include not only verification by exclusion of falsifiable hypotheses, but also rigorous implementation of the formal and informal logic that has been endorsed by scientists for over two millennia [1] [2]. Self-styled quasicrystallographers claim (e.g. [3] p. 82) that the diffraction observed in quasicrystals is Bragg diffraction. The claim might have either a weak or a strong meaning, though they evidently mean it in the strong sense. The weak sense is that the diffraction is due to a 3-dimensional scatterer rather than a 2 -dimensional grating. When a reflection grating is rotated by a small angle $\alpha$, the diffracted beam rotates specularly by $2 \alpha$; whereas the diffraction from a 3-D crystal switches sharply on and off as it rocks about the Bragg condition. In this strong sense, diffraction is described by Bragg's law: at wavelength $\lambda$, light is re- 
flected from regular interplanar spacings of width $d$ at the Bragg angle $\theta$ (the complement to the angle of incidence) which is constrained such that $n \lambda=$ $2 d \sin (\theta)$, where $n$ represents the diffraction order. This law describes diffraction in crystals. Quasicrystals provided a new and unique type of diffraction.

\section{Quasicrystal Diffraction}

\subsection{Diffractive Order $n$}

In crystals, the order is positive integral: $n=0,1,2,3 \cdots$; whereas in the quasicrystal; the order is represented in powers of the golden section $\tau^{m}$, where $\tau=\left(1+5^{1 / 2}\right) / 2$ and $m$ is positive or negative, $m=-\infty,-1,0,1,2,3 \cdots$ corresponding to $n=0,1 / \tau, 1, \tau, \tau^{2}, \tau^{3} \cdots$. The quasi-Bragg law is a new law in physics: $\tau^{m} \lambda=2 d^{\prime} \sin \left(\theta^{\prime}\right)$, where the apostrophes indicate compromise superpositions of many Bragg values. The geometric series is a special case of the Fibonacci sequence: in the former case, the ratio between successive terms is constant; in the latter case the ratio oscillates about $\tau$. Such oscillations are not observed in the quasicrystal diffraction pattern.

\subsection{Quasiperiodicity}

The discovery of Shechtman et al. [4] was entitled "a metallic phase with long range order an no translational symmetry”. Imaging showed multiple interplanar spacings irregularly jumbled. Bragg's specific $d_{h k l}$ for an indexed beam (hkl) is therefore neither unique in the quasicrystal nor periodic, so the wonder that has to be explained is how the diffraction due to such a structure can be sharp. In the following discussion, we show precisely how that occurs and how the model is verified by measurement. Meanwhile neither $n$ nor $d$ obey Bragg's law.

\subsection{The Quasi-Bragg Angle}

Since $d$ is not unique, the Bragg angle is not defined and does not obey Bragg's law either. However, we will show how the quasi-Bragg angle $\theta^{\prime}$ is calculated, and it is certainly not the Bragg angle. There is no Bragg diffraction.

\subsection{What Do We Know?}

Given the composition $\mathrm{Al}_{6} \mathrm{Mn}$, we know that $A l$ has the atomic number 13 and Mn 25. The scattering power for electrons used in transmission electron microscopy is four times greater for the transition metal. In phase-contrast optimum defocus [5] Mn is readily specified and located [3] [6] [7]. The image demonstrates a hierarchic icosahedral structure that is four tiers deep and apparently expansive indefinitely. Every atom is specified, located and measured for size.

The unit cell is edge sharing. This results in the 13 atom unit cell having the stoichiometry $\mathrm{Al}_{6} \mathrm{Mn}$ (Figure 1). It is extremely dense owing to the precise ratio of the central $\mathrm{Mn}$ atom to outer $\mathrm{Al}$ atoms. All diatomic quasicrystals share the same ratio.

Knowing the structure, we can simulate the diffraction pattern; but it is necessary first to correct indexation. 

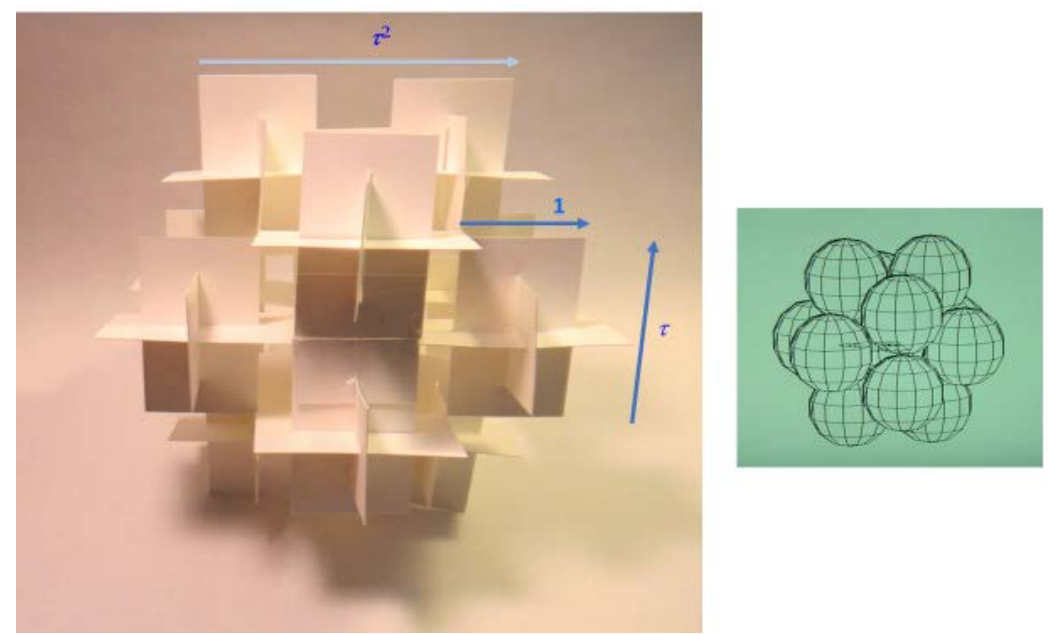

Figure 1. Dense, icosahedral, unit cell (right) and dimensions of the logarithmically periodic structure (left) with stretching factor $\tau^{2}$. This is the ratio of the edge of the icosahedral cluster to the edge of the unit cell.

\subsection{Indexation}

The stereogram of principal axes of the icosahedral structure is 3-dimensional, in geometric series, simple, and complete [8] [9]. So also are the principal diffraction planes that are normal to the axes. Following William of Ockham, dimensions should not be multiplied without necessity. Inventing dimensions does not solve physical problems ${ }^{1}$. All of the beams in the original data [4] have been indexed on this basis ([6] and refs.) and their quasi-structure factors (QSFs) calculated [3] [6] [7] and summarized as follows.

\subsection{Quasi-Structure Factors (QSFs)}

The sites of atoms and cell centers in icosahedral clusters are known [7] [9] [10], including sites in higher order $p$ of supercluster, where indices multiply by $\tau^{2 p}$ :

$$
\begin{aligned}
\text { Unit cell }\left(r_{u}\right): & \text { Mn } \\
\text { Al } & 1 / 2( \pm \tau, 0,0) \\
&
\end{aligned}
$$

and

Cell or cluster centers $\left(r_{c c}\right) \quad 1 / 2\left( \pm \tau^{2}, 0, \pm \tau\right), 1 / 2\left(0, \pm \tau, \pm \tau^{2}\right), 1 / 2\left( \pm \tau, \pm \tau^{2}, 0\right)$,

The QSF formula is adapted from classical crystallography with two differences:

$$
F_{h k l}=\sum_{i=1}^{\text {all atoms }} f_{i} \cos \left(2 \pi \cdot c_{s}\left(\overline{h_{h k l}} \cdot \overline{r_{i}}\right)\right)
$$

Firstly, because the diffraction is sharp in spite of multiple interplanar spacings $d$, a coherence factor $c_{s}$ is inserted. Its value will be derived analytically below. Initially the factor is used as a scanned variable (Figure 2) to illustrate the ${ }^{1}$ Crystallographers know that the hexagonal close packed structure is sometimes indexed with four digits; sometimes with three. Equally they know that the structure is 3-dimensional $\mathrm{R}^{3}$-in this it is like the quasicrystal. 
variance of quasi-Bragg diffraction from Bragg diffraction in crystals.

Secondly, because the unit cells are not periodic as in crystals, the summation is made over all atoms in the quasicrystal; not just the unit cell. The summation is taken in two steps: over the unit cell and cluster, and iteratively over the superclusters in hierarchic order $p$. Write the vector from the origin to each atom in a cluster $\overline{r_{c l}}$ as the sum of a unit cell vector $\overline{r_{u}}$, with a vector to the cell centers in the cluster $\overline{r_{c c}}: \overline{r_{c l}}=\overline{r_{c c}}+\overline{r_{u}}$. Then since

$$
\sum_{i}^{\text {Ncluster }} \exp \left(\overline{h_{h k l}} \cdot \overline{r_{c l}}\right)=\sum_{i}^{12} \exp \left(\overline{h_{h k l}} \cdot \overline{r_{c c}}\right) \times \sum_{i}^{13} \exp \left(\overline{h_{h k l}} \cdot \overline{r_{u}}\right)
$$

with corresponding summations over unit cell sites and cell centers, and knowing that $N_{\text {cluster }}=N_{c c} \cdot N_{u}$, the QSF for the cluster may be calculated:

$$
F_{h k l}^{\text {cluster }}=\sum_{i=1}^{12} \cos \left(2 \pi \cdot c_{s}\left(\overline{h_{h k l}} \cdot \overline{r_{c c}}\right)\right) \cdot F_{h k l}^{\text {cell }}
$$

and repeating iteratively over superclusters by using the known stretching factor $\tau^{2 P}:$

$$
F_{h k l}^{p}=\sum_{i=1}^{\text {all atoms }} \cos \left(2 \pi \cdot c_{s}\left(\overline{h_{h k l}} \cdot \tau^{2 p} \overline{r_{c c}}\right)\right) \cdot F_{h k l}^{p-1}
$$

The example in the figure is for the simple geometric series, but all beams in the original data [4] have been calculated to match. All structure factors, i.e. at the Bragg condition $c_{s}=1$, are zero. In the quasicrystal there is no Bragg diffraction: all beams peak at the quasi-Bragg condition $c_{s}=0.894$. As we shall see, this value is the result of harmonization of the incident, sine wave probe with the aperiodic, hierarchic structure.

\subsection{Quasi-Bloch Waves}

In crystals, Bloch waves [11] [12] can be observed as lattice images observed, for example, in the two beam Bragg condition. The waves occur as interference between incident and reflected waves. Two are simulated as the red trace for the quasicrystal (Figure 3 with $c_{s}=0.894$ ), for comparison with the blue, anharmonic, (100), pseudo-Bragg condition, $c_{s}=1$.

The quasi-Bloch wave harmonizes with the hierarchic structure; the pseudo-Bragg wave does not diffract.

\subsection{Principal Planes}

The golden triad contains three orthogonal golden rectangles. There are three principal planes, one for each dimension of the golden triad (Figure 4). The triad scales within the hierarchy for each order of unit cell, cluster and supercluster. The listing of interplanar spacings illustrates the origin of the geometric series in the quasicrystal diffraction pattern.

For each order of cluster, golden triads mark principal planes that locate subcluster centers at corners. These centers operate as principal scatterers for the hierarchic structure. The diffraction occurs by reflection between hierarchic 


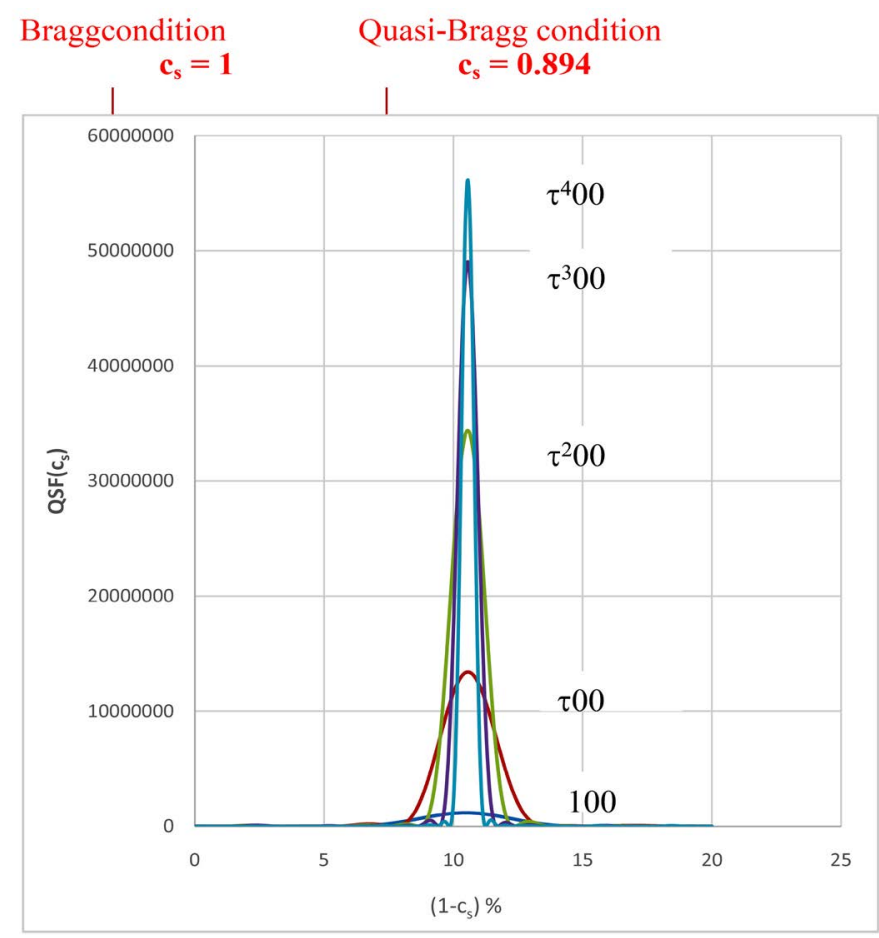

Figure 2. A selection of QSF displays obtained by scanning $c_{s}$. Indexed lines are shown for an $\mathrm{i}-\mathrm{Al}_{6} \mathrm{Mn}$ supercluster order $2\left(\sim 10^{4}\right.$ atoms). The lines peak consistently when $c_{s}=0.894$ [6] with similar intensities (FWHM). By contrast QSFs for the Bragg condition $\left(c_{s}=1\right.$ and $n=0,1,2,3 \cdots)$ are unregulated, with more or less random QSF structures, spread out and very weak. All structure factors at the Bragg condition are $\sim$ zero. There is no Bragg diffraction.

\section{Quasi-Bloch wave, $c_{s}=0.894$, harmonic}

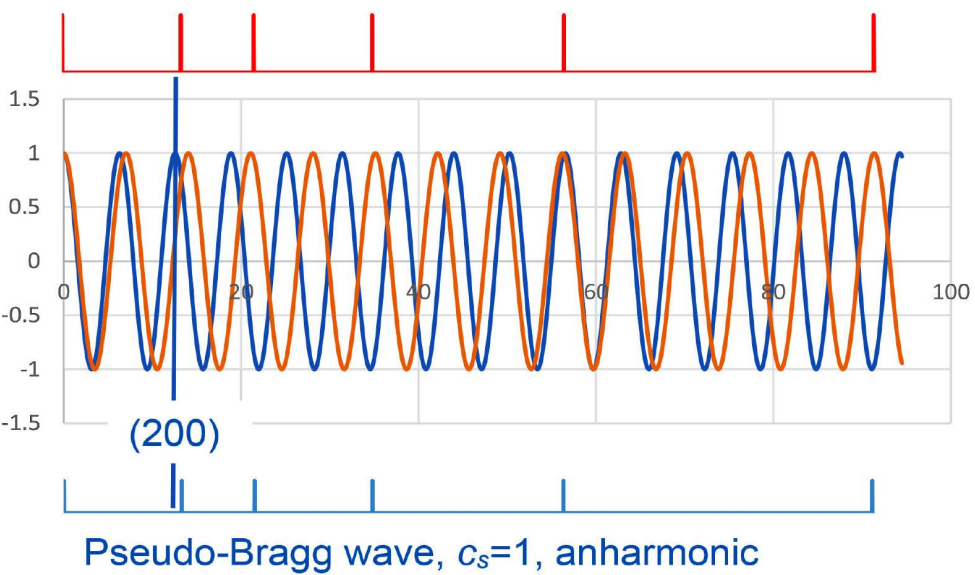

Figure 3. Plotted across principal planes (red upper grid) in geometric series in the hierarchic structure, are a red quasi-Bloch wave $\left(c_{s}=0.894\right)$ and a blue pseudo-Bragg wave $\left(c_{s}=1\right)$. The former is harmonic consistent with coherent diffraction; the latter is anharmonic and does not diffract. The metric harmonizes the quasi-Bloch wave with the hierarchic structure. The long vertical blue would mark the Bragg (200) line if it were allowed. Notice that a contraction in $d$, dilates a Bloch wave; so does $c_{s}$ in $d^{\prime}=d \cdot c_{s}$ in the quasicrystal. 
centers of unit cells, clusters and superclusters. Each reflection is weighted by parallel reflections from the bodies of respective sub-clusters or sub-superclusters. That is why, in Figure 2, the line widths narrow with increasing power on the index, consistent with increasing numbers of scattering atoms. The diffraction consists in coherent scattering from hierarchic bodies of the same order.

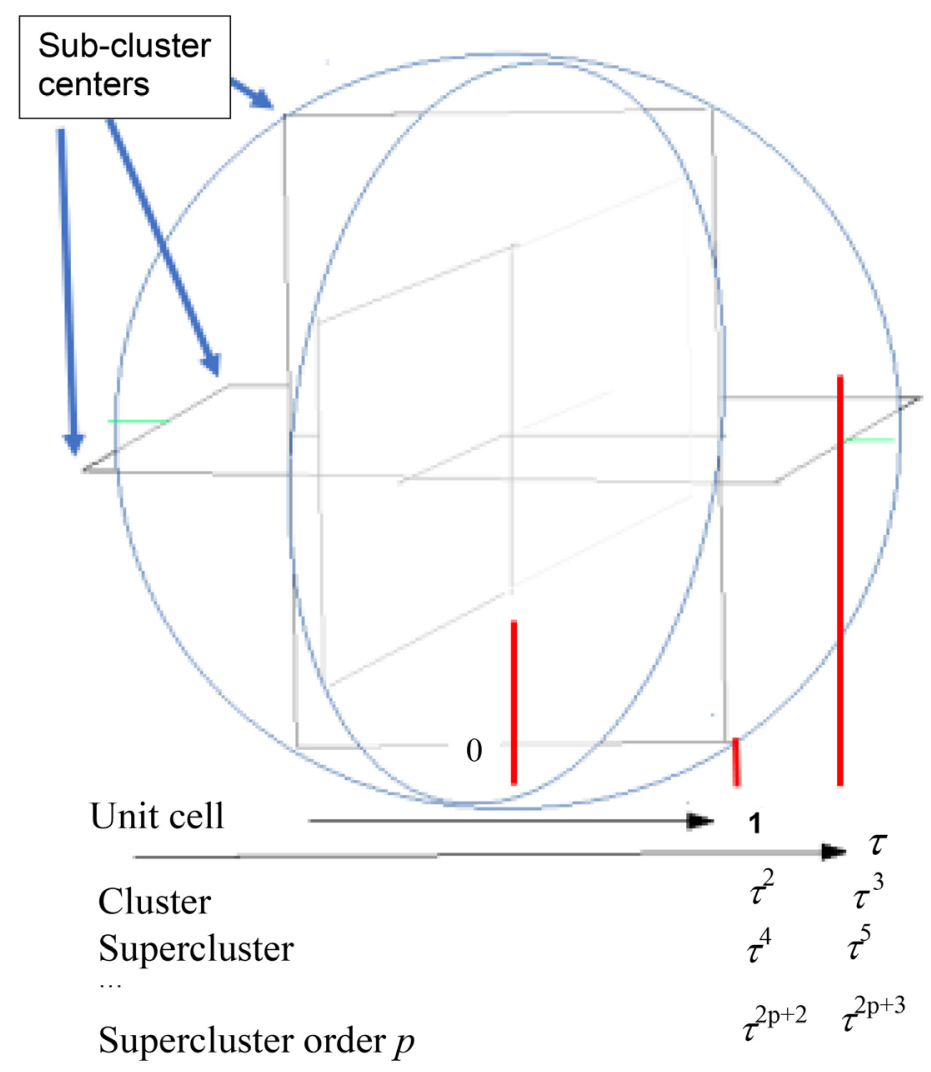

Figure 4. Principal atomic planes are located by red lines. The golden triad scales to represent hierarchic orders of: unit cell, cluster and superclusters. Notice the interplanar spacings in geometric series $0,1, \tau, \tau^{2}, \tau^{3} \cdots$ that correspond to diffraction pattern series. Coherent diffraction occurs by scattering of corresponding sub-cluster bodies centered on the corners of the golden rectangles.

\subsection{Analytic Derivation for the Metric}

The principal planes determine the coherence factor and metric (Table 1). The planes lie in geometric series. This series is irrational but a rational approximation may be constructed by the substitution of the rational fraction $3 / 2$ for $\tau$ as shown in the table. It is obvious that atoms located on the half-integral set of "coherent values" (column 7) could diffract coherently according to Bragg's law if such a structure could be constructed. It follows that, owing to the systemically derived value for the ratio $r$, atoms located on the "real" geometric-series lattice will likewise diffract coherently, but with the metric displacement $c_{s} \sim 0.894$, as in Figure 2. This fact is confirmed by the numerical QSF calculations. Coherent 
Table 1. The metric is derived approximately from principal planes (bold column 10 with underlines) as follows: Bold column 6 shows irrational real values for the geometric series and Fibonacci equivalents on the same row; coherent values are rationalized by substitution for $\tau$ in the Fibonacci equivalent (columns 1-2) by the fraction $3 / 2$ (as in the formula on the $3^{\text {rd }}$ row). The harmony in the hierarchic scattering by the incident sine wave results in coherent diffraction. The square of the ratio of Rational value/Real value, on principal planes, is close to the numerical value for $c_{s}$ given by QSF simulations from various supercluster orders (underlined in column 10). Column 10 is otherwise semi-empirical.

\begin{tabular}{|c|c|c|c|c|c|c|c|c|c|c|c|c|}
\hline \multicolumn{7}{|c|}{ Geometric series } & \multirow{3}{*}{$\begin{array}{c}\text { Irrational } \\
\text { value }\end{array}$} & \multirow{3}{*}{$\begin{array}{l}\text { Rational } \\
\text { approx. } \\
a+b^{\star} 1.5\end{array}$} & \multirow{3}{*}{ 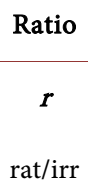 } & \multirow{4}{*}{$\begin{array}{l}r^{2} \\
\\
0\end{array}$} & \multirow{3}{*}{$\begin{array}{c}c_{s} \\
\text { estimates } \\
1-2^{\star}(1-r)\end{array}$} & \multirow{3}{*}{$\begin{array}{c}\text { QSF } \\
\text { supercluster } \\
\text { (order) }\end{array}$} \\
\hline a & & $\mathrm{b}$ & & & & & & & & & & \\
\hline & & & & & & & & & & & & \\
\hline 0 & & & & $=$ & 0 & $=$ & 0 & 0 & 0 & & 0 & \\
\hline 1 & & & & $=$ & 1 & $=$ & 1 & 1 & 1 & 1 & 1 & \\
\hline & & & $t$ & $=$ & $t$ & $=$ & 1.61803 & 1.5 & 0.927 & 0.859 & 0.854 & \\
\hline 1 & + & & $t$ & $=$ & $t^{2}$ & $=$ & 2.61803 & 2.5 & 0.955 & 0.912 & 0.91 & \\
\hline 1 & + & 2 & $t$ & $=$ & $t^{3}$ & $=$ & 4.23607 & 4 & 0.944 & 0.891 & 0.888 & \\
\hline 2 & + & 3 & $t$ & $=$ & $t^{t}$ & $=$ & 6.8541 & 6.5 & 0.948 & 0.899 & 0.896 & \\
\hline 3 & + & 5 & $t$ & $=$ & $t^{5}$ & $=$ & 11.0902 & 10.5 & 0.947 & 0.897 & 0.894 & \\
\hline 5 & + & 8 & $t$ & $=$ & $t^{6}$ & $=$ & 17.9443 & 17 & 0.947 & 0.897 & 0.894 & \\
\hline 8 & + & 13 & $t$ & $=$ & $t^{7}$ & $=$ & 29.0344 & 27.5 & 0.947 & 0.897 & 0.894 & \\
\hline 13 & + & 21 & $t$ & $=$ & $t^{8}$ & $=$ & 46.9787 & 44.5 & 0.947 & 0.897 & $\underline{0.894}$ & $\underline{\operatorname{sc}(2)}$ \\
\hline 21 & + & 34 & $t$ & $=$ & $t^{\ominus}$ & $=$ & 76.0132 & 72 & 0.947 & 0.897 & 0.894 & \\
\hline 34 & + & 55 & $t$ & $=$ & $t^{10}$ & $=$ & 122.992 & 116.5 & 0.947 & 0.897 & $\underline{0.894}$ & $\underline{\mathrm{sc}(3)}$ \\
\hline 55 & + & 89 & $t$ & $=$ & $t^{11}$ & $=$ & 199.005 & 188.5 & 0.947 & 0.897 & 0.894 & \\
\hline 89 & + & 144 & $t$ & $=$ & $t^{12}$ & $=$ & 321.997 & 305 & 0.947 & 0.897 & 0.894 & \\
\hline 144 & + & 233 & $t$ & $=$ & $t^{13}$ & $=$ & 521.002 & 493.5 & 0.947 & 0.897 & 0.894 & \\
\hline 233 & + & 377 & $t$ & $=$ & $t^{14}$ & $=$ & 842.999 & 798.5 & 0.947 & 0.897 & 0.894 & \\
\hline 377 & + & 610 & $t$ & $=$ & $t^{15}$ & $=$ & 1364 & 1292 & 0.947 & 0.897 & 0.894 & \\
\hline 610 & + & 987 & $t$ & $=$ & $t^{16}$ & $=$ & 2207 & 2090.5 & 0.947 & 0.897 & $\underline{0.894}$ & $\underline{\mathrm{sc}(6)}$ \\
\hline
\end{tabular}

$r^{*} r^{+}:\left(\because\right.$ Intensities $\left.\alpha \mathrm{QSF}^{2}\right)$ here calculated for principal planes only; \#: values simulared QSFs including ALL planess, and these match estimates; and elementary estimates: $c_{s}=1 /(1+(t-1.5))$ and $c_{s}=1 /\left(1-\left(t^{2}-2.5\right)\right)$.

diffraction occurs by the QSF selection of $d^{\prime}=d \cdot c_{s}$ and consequently of $\theta^{\prime}=q / c_{s}$ under the quasi-Bragg law. Consistent with the measured value for the lattice parameter to be discussed below, the metric $\mathcal{C}_{s}$ is the ratio of corresponding terms: (coherent value $)^{2} /(\text { real value })^{2}$. The square on the ratio is due to wave mechanics, where the intensities of the beams are-for centrosymmetric structures-squares of corresponding amplitudes. 


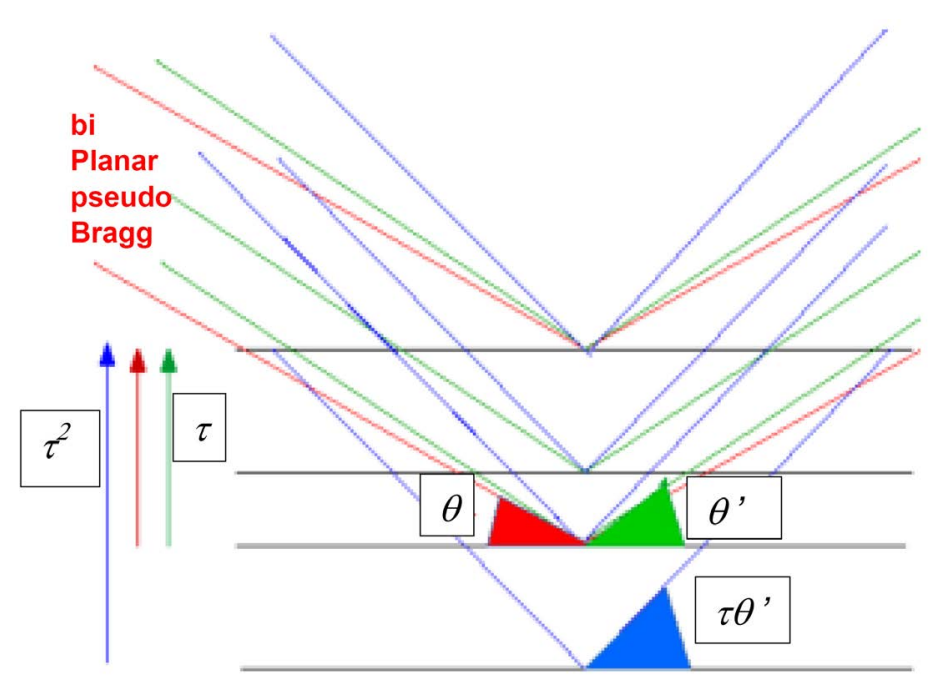

Figure 5. Models for biplanar Bragg diffraction (red rays) compared with multiplanar quasi-Bragg diffraction (green rays for first order; blue for second order), where all atoms scatter. Notice the multiplanar quasi-Bragg angle $\theta^{\prime}$ is larger than the corresponding biplanar pseudo-Bragg angle $\theta$.

\subsection{The Model for Quasicrystal Diffraction}

All atoms scatter. Whereas the uniqueness of $d$ and its periodicity forces Bragg diffraction in crystals to reflect as biplanes, quasiperiodicity forces multiplanar reflections in hierarchic quasicrystals (Figure 5). Their superposition is described by the QSFs.

On the well-known model, the path difference between two reflections from adjacent Bragg planes is equal to the wavelength of the light, with $c_{s}=1$. QSFs imitate the corresponding interference of the quasi-Bragg rays from multiple planes at the quasi-Bragg condition (i.e. when $c_{s}=0.894$ ). Notice that the filled green quasi-Bragg angle is larger than the corresponding filled red Bragg angle.

In Bragg diffraction, when $d$ contracts $\theta$ dilates; in quasi-Bragg diffraction, when $c_{s}$ (and $d$ ) contract, $\theta^{\prime}$ dilates. The dilatation is enforced by a constructive interference requirement for harmonic reflections. Actually, the better model for quasicrystal diffraction is shown in Figure 4: the diffraction consists in coherent scattering from sub-cluster centers rather than Bragg biplanes.

\section{Summary}

The correlating roles of quasi-structure factors and quasi-Bragg law are summarized in Table 2, where they are compared with Bragg diffraction in crystals. The QSF simulates the metric, the compromise interplanar spacing and indexation. With these parameters, the quasi-Bragg law measures the compromise quasi-Bragg angle. The summary shows how the measured lattice parameter is corrected for metric and indexation. The result is consistent in both structure and diffraction: they are measured, and verified.

From these measurements the reciprocal lattice can be derived [3] [6] [13]. The original data show two superposed lattices owing to subgroups in the 
Table 2. Comparison of parameters used to describe Bragg diffraction with parameters for quasi-Bragg diffraction in hierarchic structures. The quasi-structure factor is used to calculate the metric $c_{s}$, the lattice parameter $a$ and the compromise inter planar spacing $d$. The quasi-Bragg Law is used to calculate the compromise quasi-Bragg angle $q_{h k l}^{\prime}=q_{h k l} / c_{s}^{\prime}$. The lattice parameter, that previously has been measured under Bragg assumptions, is corrected for $c_{s}$ and indexation. The measurement is verified by consistency between atomic size, diffraction, and structure.

\begin{tabular}{ccc} 
Comparison of Bragg parameters in crystals, with quasi-Bragg parameters in quasicrystals \\
\hline Bragg & \multicolumn{1}{c}{ Quasi-Bragg } & Comment \\
\hline$n=2 d \sin (q) / \lambda$ & $\tau^{m}=2 d^{\prime} \sin \left(\theta^{\prime}\right) / \lambda$ & Harmonic laws \\
$F_{h k l}=\sum f_{i} \cos \left(2 \pi h_{h k l} \cdot r_{i}\right)$ & $F_{h k l}^{\prime}=\sum f_{i} \cos \left(2 \pi c_{s}\left(h_{h k l} \cdot r_{i}\right)\right)$ & Give us $\theta^{\prime}=\theta / c_{s}$ \\
including iteration & Structure factors \\
$d=a / h$ & $d^{\prime}=a c_{s} h$ & Give $c_{s}$ a and $d^{\prime}=d \cdot c_{s}$ \\
& $a=0.205 \tau c_{s} \mathrm{~nm}$ & $\theta^{\prime}=\theta / c_{s}$ \\
$a^{*}=2 p / a$ & Measured lattice parameter \\
& & $a \approx$ Diameter of $\boldsymbol{A l}$ \\
& & Measured and verified
\end{tabular}

n: Bragg order; $m$ : Quasi-Bragg order; $d$ : Bragg interplanar spacing; $\theta$. Bragg angle; $\lambda$ : wavelength; $\tau$ : golden section; prime: quasi-Bragg compromise; $F$ : Structure factors; $f_{i}$ atomic scattering factor for atom $i ; c_{s}$ metric; $r_{i}$ atom position; $h_{h k l}$ : plane normal for indices $h, k, l$; a: lattice parameter (cubic) $\sim A l$ diameter; reciprocal: lattice vector $a^{*}=2 \pi / a$.

icosahedral point group symmetry. The dual lattice is also consistent with the analysis. Moreover, the hierarchic translational symmetry is the obvious reason for the "long range order" [4] in what are typically called "quasiperiodic solids".

Moreover, the QSF simulations, when combined with harmonic analysis, demonstrate that the diffraction occurs on a scale that is a contraction of the irrational hierarchic scale of the structure. In consequence, all structural measurements that are derived from the diffraction pattern, are subject to the measured metric.

In particular, the fact that the lattice parameter was previously tentatively measured by wrongly assuming Bragg's law [14] [15], now has a corrected value (Table 2) equal to both the diameter of the $\mathrm{Al}$ atom and the width of the unit cell. This is necessary verification for the consistent theory.

\section{Conclusion}

The analytic metric completes the union of structure with diffraction. The hierarchic structure transforms the plane incident wave into geometric space. It is time to append the context. In comparatively recent times Senechal wrote for the American Mathematical Society a paper titled, "What is a quasicrystal?" The paper began, "The short answer is no one is sure" [16]. This was not true; the analytic metric shows why. The long path has been delayed by stray paths and some of them can be listed. Dimensions should not be multiplied without necessity-they do not solve physical problems. Mathematical axioms are chosen; physical hypotheses require verification (or more strictly falsification). While 
theorems must be consistent with chosen axioms; consistency is not required in physical hypotheses because they are equal as "logically true" until one is "falsified" (referee preference is no ground for refusing publication). Categories have been erroneously applied. For example, the sub-editor of Acta Crystallographica wrote that you don't measure the lattice parameter, "You just have to choose ' $d_{h}$ '” ([3] p. 82). This is like saying, "You don't measure the speed of light; you choose it," or, "You don't calculate the speed of light; you choose it." He continued by writing, "Bragg's equation cannot be applied if we do not know how to handle the term $d_{h}$." He chose an untested law that never applies. It is not normal to comment on necessities of physical practice, nor on formal and informal logic; but 37 years are a long delay.

\section{Conflicts of Interest}

The author declares no conflicts of interest regarding the publication of this paper.

\section{References}

[1] Popper, K.R. (1959) The Logic of Scientific Discovery. ßBasic Books, Inc., New York. https://doi.org/10.1063/1.3060577

[2] Popper, K.R. (1982) Quantum Theory and the Schism in Physics.

[3] Bourdillon, A.J. (2012) Metric, Myth and Quasicrystals. UHRL, San Jose.

[4] Shechtman, D., Blech, I., Gratias, D. and Cahn, J.W. (1984) Physical Review Letters, 53, 1951-1953. https://doi.org/10.1103/PhysRevLett.53.1951

[5] Bursill, L.A. and Peng, J.L. (1985) Nature, 316, 50-51. https://doi.org/10.1038/316050a0

[6] Bourdillon, A.J. (2019) Journal of Modern Physics, 10, 624-634. https://doi.org/10.4236/jmp.2019.106044

[7] Bourdillon, A.J. (2009) Quasicrystals and Quasi Drivers. UHRL, San Jose.

[8] Bourdillon, A.J. (2013) Micron, 51, 21-25. https://doi.org/10.1016/j.micron.2013.06.004

[9] Bourdillon, A.J. (2016) Journal of Modern Physics, 7, 43-50. https://doi.org/10.4236/jmp.2016.71005

[10] Bourdillon, A.J. (2016) Journal of Modern Physics, 7, 1558-1567. https://doi.org/10.4236/jmp.2016.712142

[11] Bourdillon, A.J. (2011) Logarithmically Periodic Solids. Nova Science, New York.

[12] Hirsch, P., Howie, A., Nicholson, R.B., Pashley, D.W. and Whelan, M.J. (1977) Electron Microscopy of Thin Crystals. Krieger, New York.

[13] Bourdillon, A.J. (2019) The Analytic Metric in Quasicrystals, and the Reciprocal Lattice. https://www.youtube.com/watch?v=v011ZAUimq4

[14] Bourdillon, A.J. (1987) Philosophical Magazine Letters, 55, 21-26. https://doi.org/10.1080/09500838708210435

[15] Tsai, A.P. (2008) Science and Technology of Advanced Materials, 9, 1-20.

[16] Senechal, M. (2006) Notices to the American Mathematical Society, 3, 886-887. 